\title{
Supra-treatment threshold neonatal jaundice: Incidence in HIV-exposed compared to non-exposed neonates at Queen Elizabeth Central Hospital in Blantyre, Malawi
}

\section{WNakanga, P Patel, S Panjwani, N Kennedy, K Kawaza}

Department of Paediatrics \& Child Health, College of Medicine, University of Malawi, Blantyre, Malawi

Correspondence to: Wisdom Nakanga

E-mail: wisdomnakanga@gmail.com

Abstract

\section{Introduction}

Jaundice is the yellowish pigmentation of the skin, sclera, and mucous membranes resulting from bilirubin deposition. Children born to mothers with HIV are more likely to be born premature, with low birth weight, and to become septic - all risk factors for neonatal jaundice. Further, there has been a change in the prevention of mother-to-child transmission (PMTCT) of HIV guidelines from single-dose nevirapine to a six-week course, all of which theoretically put HIV-exposed newborns at greater risk of developing neonatal jaundice.

\section{Aim}

We carried out a study to determine the incidence of severe and clinical neonatal jaundice in HIV-exposed neonates admitted to the Chatinkha Nursery $(\mathrm{CN})$ neonatal unit at Queen Elizabeth Central Hospital (QECH) in Blantyre.

\section{Methods}

Over a period of four weeks, the incidence among non-exposed neonates was also determined for comparison between the two groups of infants. Clinical jaundice was defined as transcutaneous bilirubin levels greater than $5 \mathrm{mg} / \mathrm{dL}$ and severe jaundice as bilirubin levels above the age-specific treatment threshold according the QECH guidelines. Case notes of babies admitted were retrieved and information on birth date, gestational age, birth weight, HIV status of mother, type of feeding, mode of delivery, VDRL status of mother, serum bilirubin, duration of stay in $\mathrm{CN}$, and outcome were extracted.

\section{Results}

Of the 149 neonates who were recruited, 17 (11.4\%) were HIV-exposed. One $(5.88 \%)$ of the $17 \mathrm{HIV}$-exposed and $19(14.4 \%)$ of $132 \mathrm{HIV}$ non-exposed infants developed severe jaundice requiring therapeutic intervention $(p=0.378)$. Eight $(47 \%)$ of the HIV-exposed and $107(81 \%)$ of the non-exposed neonates had clinical jaundice of bilirubin levels greater than $5 \mathrm{mg} / \mathrm{dL}(\mathrm{p}<0.001)$.

\section{Conclusions}

The study showed a significant difference in the incidence of clinical jaundice between the HIV-exposed and HIV-non-exposed neonates. Contrary to our hypothesis, however, the incidence was greater in HIVnon-exposed than in HIV-exposed infants.

\section{Introduction}

Jaundice is the yellowish pigmentation of the skin, sclera and mucous membranes. ${ }^{1}$ The yellow colour results from the accumulation of bilirubin pigment in the skin. ${ }^{2}$ Neonatal jaundice or neonatal hyperbilirubinaemia refers to jaundice that is present at birth or appears at any time during the neonatal period. $^{2}$ In neonates, hyperbilirubinaemia is detected when the serum bilirubin levels become greater than $5 \mathrm{mg} / \mathrm{dL}$ and is termed clinical jaundice. ${ }^{3}$ Jaundice can be physiological or pathological. ${ }^{4}$

Physiological jaundice is a common problem in term and preterm infants during the first week after birth and is the result of increased bilirubin production from the breakdown of foetal red blood cells combined with transient limitations in the conjugation of bilirubin by the immature neonatal liver. ${ }^{2}$ For most of these infants, jaundice is mild and resolves without treatment. More than $50 \%$ of normal newborn, and $80 \%$ of preterm infants, have some jaundice. ${ }^{5}$

A small group of infants develop pathological jaundice. This may be an exaggeration of normal physiologic processes or may be an indicator of underlying disorders. ${ }^{6}$ Pathological jaundice usually appears in the first 24 hours or after the first week of life, and the list of differential diagnoses include haemorrhage, sepsis, and congenital infections, such as toxoplasmosis, rubella, cytomegalovirus, herpes, and syphilis. $^{2}$

Risk factors of neonatal jaundice include low birth weight, prematurity, ABO incompatibility, and sepsis. ${ }^{3}$ In Malawi, $10 \%$ of neonates are born to HIV seroreactive mothers. ' Several studies have found very high rates of prematurity, low birth weight, and very low birth weight (all risk factors for neonatal jaundice) in the infants of HIVpositive mothers. ${ }^{8,9}$ In addition, other perinatal infections that predispose neonates to jaundice, such as hepatitis $\mathrm{B}$ and C, syphilis, toxoplasmosis, cytomegalovirus, herpes simplex virus, and tuberculosis exhibit high co-morbidity with HIV. ${ }^{10}$ Nevirapine has been known to cause hepatic inflammation and therefore is associated with increased levels of serum bilirubin. ${ }^{10,11}$ The new prevention of motherto-child transmission (PMTCT) guidelines from single-dose nevirapine prophylaxis to a six-week course in newborns, ${ }^{10}$ means that, in theory, the risk of developing clinical jaundice in HIV-exposed neonates is increased.

Non-invasive techniques for bilirubin measurement that correlate with serum bilirubin levels, such as with the use of a transcutaneous bilirubinometer, may be used to screen infants, but determination of serum bilirubin level is indicated in patients with elevated age-specific transcutaneous measurement, progressing jaundice, or risk for either haemolysis or sepsis. ${ }^{2}$ Severe jaundice is defined as clinical jaundice and serum bilirubin levels above treatment threshold levels for gestational age and postpartum age. If the bilirubin level is greater than the threshold value, the neonate is started on phototherapy. ${ }^{12}$ The levels of bilirubin, which are determined as supra-threshold and require treatment, are different according to the days of life and whether the neonate is healthy or preterm, as summarized in Table $1 .^{12}$

Table 1: Treatment thresholds for neonatal jaundice depending on day of life and clinical status of neonate ${ }^{2}$

\begin{tabular}{rcc} 
Day of life & $\begin{array}{c}\text { Healthy term baby } \\
(\mathbf{m g} / \mathbf{d L})\end{array}$ & $\begin{array}{c}\text { Preterm, low birth } \\
\text { weight, sick }(\mathbf{m g} / \mathbf{d L})\end{array}$ \\
\hline Day 1 & $\begin{array}{c}\text { Treat any visible } \\
\text { jaundice with } \\
\text { phototherapy }\end{array}$ & $\begin{array}{c}\text { Treat any visible } \\
\text { jaundice with } \\
\text { phototherapy }\end{array}$ \\
Day 2 & 15 & 13 \\
Day 3 & 18 & 16 \\
Day 4 and after & 20 & 17 \\
\hline
\end{tabular}


Jaundice-if untreated or if treatment thereof is delayedcan lead to significant neurological sequelae. These include movement disorders like athetoid cerebral palsy and dystonia, auditory dysfunction, auditory neuropathy (ANSD), oculomotor impairments (nystagmus, strabismus, impaired upward or downward gaze, and/or cortical visual impairment) and other disorders such as dental enamel hypoplasia/ dysplasia of the deciduous teeth, gastroesophageal reflux, and impaired digestive function. ${ }^{2}$

While there is a correlation between the risk factors for neonates to become jaundiced and the risks and comorbidities that HIV-exposed neonates face, few studies have compared the incidence of neonatal jaundice between HIVexposed and HIV-non-exposed neonates. The hypothesis of this study was that the incidence of jaundice may be higher in HIV-exposed neonates than in HIV-non-exposed neonates.

\section{Methods}

\section{Study population, sample size, and study setting}

This prospective cohort study was conducted at the Gogo Chatinkha Nursery $(\mathrm{CN})$ neonatal unit at Queen Elizabeth Central Hospital over a four-week period. The admission criteria for $\mathrm{CN}$ includes those neonates who are premature, of low birth weight, or are exibiting any danger signs in a neonate, for example, dyspnoea or poor colour. The study population comprised neonates admitted to $\mathrm{CN}$. Only neonates who met the inclusion criteria were included in the study after consent was obtained from their mothers.

The inclusion criteria were that newborn babies had to be free from jaundice and admitted to $\mathrm{CN}$. The following were not eligible for the study: neonates who already had jaundice, neonates with significant congenital anomalies, and neonates of mothers whose HIV statuses were not known.

The study participants were recruited using stratified sampling. Two groups of eligible neonates whose mothers were HIV-positive and HIV-negative were identified. A random sample of 69 babies was drawn from each group. The calculated sample size of 138 (69 exposed and 69 nonexposed) babies was generated using Epi info version 3.5, with a confidence level of $90 \%$, power of $80 \%$, exposed to non exposed-ratio of 1:1, risk ratio of 2.31, and an odds ratio of 3 . However, we did not reach our sample size, as only 17 HIV-exposed neonates were recruited. 149 neonates were recruited in order to increase the number of HIV-exposed neonates in the study, as not many HIV-exposed neonates were admitted to $\mathrm{CN}$ during the data collection period. Data on the sampled babies were collected using self-administered questionnaires, which were provided to the mothers. Data were analysed using Epi Info 3.5 software.

\section{Ethical considerations}

Ethical approval was granted by the College of Medicine Research and Ethics Committee (COMREC). Informed written consent was sought from the mothers of the neonates. Before consent was obtained, the mothers were assured of confidentiality. Study subjects were each assigned unique identification numbers.

\section{Data collection}

Once the mothers consented, details of the mother and baby were recorded on data collection forms. Babies had wrist bands on which their mothers' names were written. These wristbands were checked daily before taking any measurements from the babies.

\section{Data management}

Bilirubin levels were measured using a transcutaneous bilirubinometer, which was placed on the sternum and forehead of each baby, and the highest reading was recorded. Axillary temperatures were also measured using a thermometer. If a study subject's level of bilirubin was above the accepted treatment threshold, the investigators (three of whom were fourth-year medical students at the time of data collection) discussed the case with the doctor on duty for information about the treatment plan for the baby. The babies who developed jaundice were started on phototherapy. The measurements for temperature and bilirubin were taken every day for the duration of stay of the baby in $\mathrm{CN}$. If pyrexia or changes in condition of the study patients was noted by the investigators, this information was immediately relayed to the clinicians responsible for patient care. Babies who developed jaundice, as well as those who did not, were monitored throughout their duration of stay in $\mathrm{CN}$.

\section{Data analysis}

Microsoft Excel 2011 and Epi info 3.5 were used to record, store, and analyse the data that were collected.

\section{Results}

The total number of admitted neonates from 11 September 2013 to 9 October 2013 was 251. Of the 251 neonates admitted to $\mathrm{CN}, 149$ neonates were recruited into the study. The prevalence of HIV among mothers giving birth at QECH was $13.8 \%$. Sixty-one percent $(91 / 149)$ of the neonates recruited into the study were males. Ten mothers $(6.3 \%)$ who gave consent had not been tested for HIV, hence children born to these mothers were excluded from the study.

Table 2: Demooraphic characteristics of neonates born to HIVpositive and HIV-negative mothers

\begin{tabular}{cc} 
HIV-positive & HIV-negative \\
$n(\%)$ & $n(\%)$ \\
\hline
\end{tabular}

Gender

$\begin{array}{ccc}\text { Male } & 10(58.8) & 81(61.4) \\ \text { Female } & 7(41.2) & 51(38.6)\end{array}$

Gestational age

Preterm (<37wks)

$10(58.8)$

$46(34.8)$

Term $(37 \leq$ gestation $\leq 40)$

$7(41.2)$

$86(65.2)$

Birth weight

$\begin{array}{lll}<2500 \mathrm{~g} & 13(76.5) & 51(38.6) \\ \geq 2500 \mathrm{~g} & 4(23.5) & 81(61.4)\end{array}$

VDRL of mother

$\begin{array}{ccc}\text { Negative } & 2(11.8) & 16(12.1) \\ \text { Not tested } & 15(88.2) & 116(87.9)\end{array}$

Mode of delivery

$\begin{array}{ccc}\text { SVD } & 12(70.6) & 67(50.8) \\ \text { CS } & 4(23.5) & 50(37.9) \\ & 1(5.9) & 15(11.4)\end{array}$

Other (e.g., breech, vacuum) $\quad 1$ (5.9) 15 (11.4)

VDRL = Venereal Disease Research Laboratory (test) $S V D=$ Spontaneous vertex delivery $\mathrm{CS}=$ Caesarean section 
Over $75 \%$ of neonates admitted to $\mathrm{CN}$ developed clinical jaundice (Table 3). Over half (64\%) of the neonates with clinical jaundice were male, while the remainder $(36.0 \%)$ were female. Gender was significantly associated with clinical jaundice $(\mathrm{p}=0.033)$.

About $80 \%$ of neonates had low birth weight. Seventy percent of neonates with normal birth weight (birth weight $\geq 2500 \mathrm{~g}$ ) developed clinical jaundice. However, birth weight was not significantly associated with clinical jaundice ( $p$ $=0.140)$. About half of the HIV-exposed neonates and the majority $(81 \%)$ of the HIV-non-exposed neonates developed clinical jaundice (Figure 1). HIV non-exposure was significantly associated with clinical jaundice $(\mathrm{p}<0.001)$. Over $70 \%$ of full-term neonates and about $80 \%$ of preterm neonates developed clinical jaundice.

Table 3: Associations between clinical jaundice and demographic characteristics of study participants

\begin{tabular}{|c|c|c|c|}
\hline & $\begin{array}{c}\text { Clinical } \\
\text { jaundice } \\
\text { (> } 5 \mathrm{mg} / \mathrm{dL}) \\
n(\%)\end{array}$ & No jaundice & $\begin{array}{c}\text { Chi-squared } \\
\text { p-value }\end{array}$ \\
\hline \multicolumn{4}{|l|}{ Gender } \\
\hline Female & $41(70.7)$ & $17(29.3)$ & \multirow[b]{2}{*}{0.033} \\
\hline Male & $73(80.2)$ & $18(19.8)$ & \\
\hline \multicolumn{4}{|l|}{ Gestational age } \\
\hline Preterm (< 37 weeks) & $47(79.7)$ & $12(20.3)$ & \multirow{2}{*}{0.753} \\
\hline Term ( $37 \leq$ gestation $\leq 40$ ) & $65(74.7)$ & $22(25.3)$ & \\
\hline \multicolumn{4}{|l|}{ Birth weight } \\
\hline$<2500 \mathrm{~g}$ & $52(81.3)$ & $12(18.8)$ & \multirow{2}{*}{0.140} \\
\hline$\geq 2500 \mathrm{~g}$ & $61(73.5)$ & $22(26.5)$ & \\
\hline \multicolumn{4}{|l|}{ HIV status } \\
\hline Exposed & $8(47.1)$ & 9 (52.9) & \multirow[b]{2}{*}{$<0.001$} \\
\hline Not exposed & $107(81.1)$ & 25 (18.9) & \\
\hline \multicolumn{4}{|l|}{ VDRL of mother } \\
\hline Negative & $14(77.8)$ & $4(22.2)$ & \multirow{2}{*}{0.969} \\
\hline Not tested & $101(77.1)$ & $30(22.9)$ & \\
\hline \multicolumn{4}{|l|}{ Mode of delivery } \\
\hline SVD & $65(82.3)$ & $14(17.7)$ & \multirow{3}{*}{0.246} \\
\hline CS & $36(66.7)$ & $18(33.3)$ & \\
\hline Other (eg., breech, vacuum) & $14(87.5)$ & $2(12.5)$ & \\
\hline
\end{tabular}

VDRL = Venereal Disease Research Laboratory (test)

$S V D=$ Spontaneous vertex delivery

$\mathrm{CS}=$ Caesarean section

Figure 1: Incidence of clinical jaundice according to HIV exposure $100 \%$

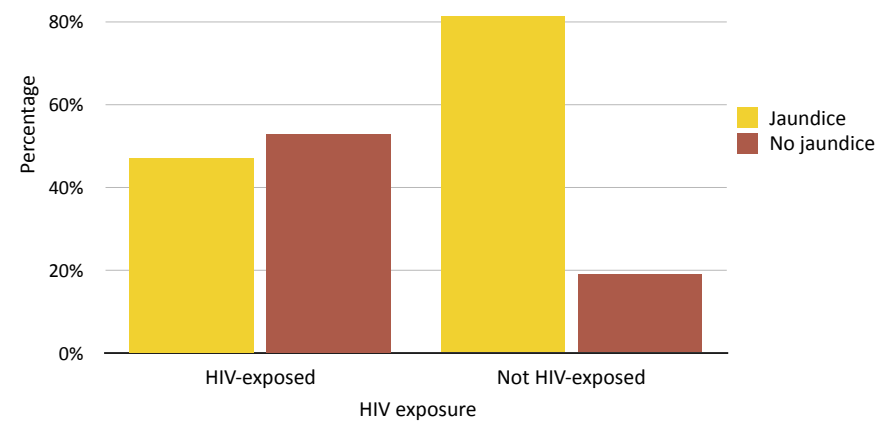

Fifteen of the 17 HIV-exposed neonates were given nevirapine, as the mothers of two HIV-exposed neonates refused nevirapine prophylaxis. Both neonates who were not given nevirapine developed clinical jaundice. Six (40\%) of the 15 neonates who were given nevirapine developed clinical jaundice.

The VDRL test was not performed on the majority of the mothers $(88 \%)$. None of the mothers who were tested were VDRL-positive. Over $75 \%$ of neonates whose mothers were not tested (VDRL test) developed clinical jaundice.

Among neonates born via spontaneous vertex delivery (SVD), over $80 \%$ developed clinical jaundice. Over $60 \%$ of neonates born via caesarean section (CS) developed clinical jaundice. Almost all neonates (88\%) born by alternative methods (breech or vacuum extraction) developed clinical jaundice. The mean bilirubin level for all neonates with and without HIV exposure peaked on day 4 (Figure 2).

Figure 2: Average bilirubin levels during the first 10 days of life

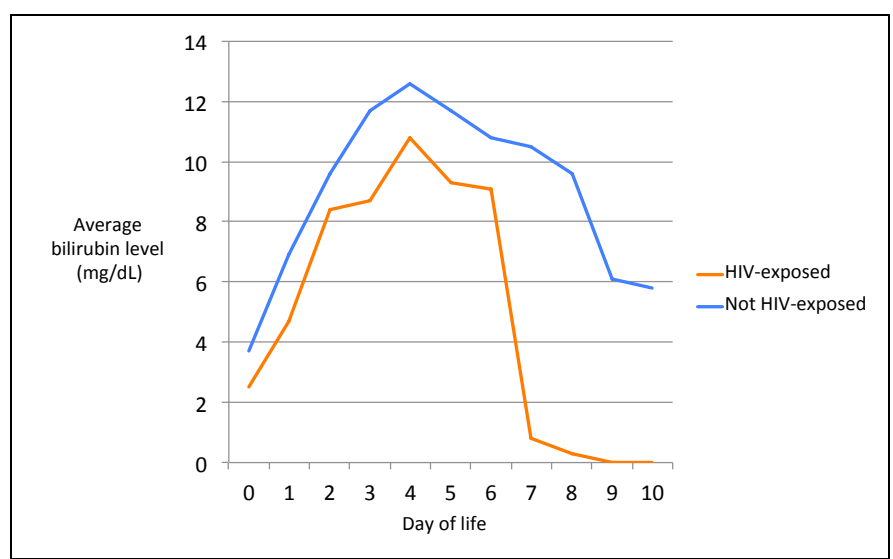

Figure 3: Incidence of severe jaundice according to HIV exposure

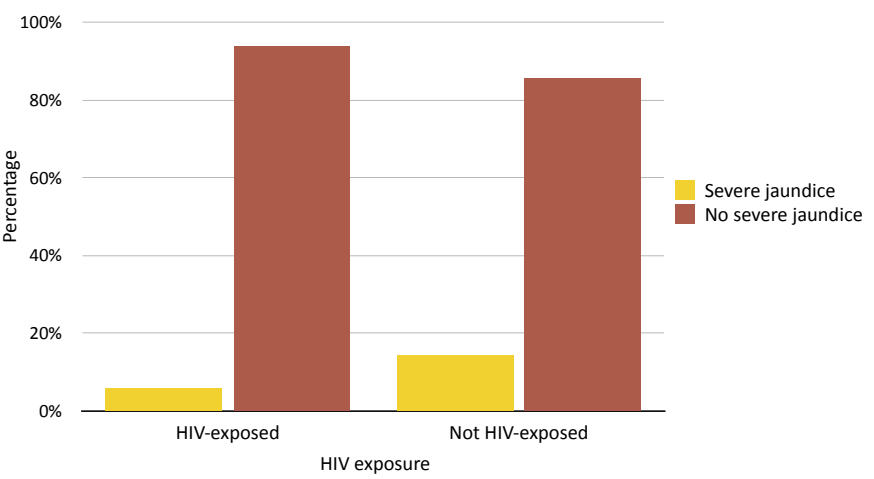

About $10 \%$ of the neonates developed severe jaundice and were put on phototherapy. Most of the neonates who developed severe jaundice were HIV-non-exposed (Figure 3). About $13 \%$ of neonates with low birth weights and $15 \%$ of neonates with normal birth weights developed severe jaundice. About $6 \%$ of HIV-exposed neonates and 14\% of HIV-non-exposed neonates developed severe jaundice. Severe jaundice was not significantly associated with HIV exposure. About $12 \%$ of term neonates and $15 \%$ of preterm neonates developed severe jaundice. Sixteen $(12.2 \%)$ of the 131 neonates whose mothers were not VDRL tested and $4(22 \%)$ of the 18 neonates whose mothers were VDRLnegative developed severe jaundice. Nine $(11.4 \%)$ of the 79 neonates born via SVD, nine $(16.7 \%)$ of the 54 neonates born via CS and $2(12.5 \%)$ of the 16 born by alternative methods (for example, breech or vacuum extraction) developed severe jaundice. Most neonates developed severe jaundice on day 3. 
The peak and fall in bilirubin levels was similar for both clinical and severe jaundice (Figure 4).

Adjusting for birth weight, mode of delivery, gestational age, gender and VDRL test, clinical jaundice was associated with HIV status $(\mathrm{p}<0.001)$ (Table 5). Neonates with HIV exposure were $90 \%$ less likely to develop clinical jaundice than neonates without HIV exposure.

The odds of clinical jaundice are 2.6 times higher among male neonates than among female neonates when other factors are controlled for.

Controlling for birth weight, mode of delivery, gestational age, gender, and VDRL status, severe jaundice was not associated with HIV status $(p=0.378)$ (Table 6).

Table 4: Association between severe jaundice and demographic characteristics of study participants

\begin{tabular}{|c|c|c|c|}
\hline & $\begin{array}{c}\text { Severe } \\
\text { jaundice } \\
\mathrm{n}(\%)\end{array}$ & $\begin{array}{l}\text { No severe } \\
\text { jaundice }\end{array}$ & $\begin{array}{c}\text { Chi-squared } \\
\text { p-value }\end{array}$ \\
\hline \multicolumn{4}{|l|}{ Gender } \\
\hline Female & $4(6.9)$ & $54(93.1)$ & \multirow{2}{*}{0.114} \\
\hline Male & $16(17.6)$ & $75(82.4)$ & \\
\hline \multicolumn{4}{|l|}{ Gestational age } \\
\hline Preterm ( $<37$ weeks) & $9(15.3)$ & $50(84.7)$ & \multirow{2}{*}{0.432} \\
\hline Term $(37 \leq$ gestation $\leq 40)$ & $11(12.4)$ & $78(87.6)$ & \\
\hline \multicolumn{4}{|l|}{ Birth weight } \\
\hline$<2500 \mathrm{~g}$ & $8(12.5)$ & $56(87.5)$ & \multirow{2}{*}{0.525} \\
\hline$\geq 2500 \mathrm{~g}$ & $12(14.5)$ & $71(85.5)$ & \\
\hline \multicolumn{4}{|l|}{ HIV status } \\
\hline Exposed & $1(5.9)$ & $16(94.1)$ & \multirow{2}{*}{0.378} \\
\hline Not exposed & $19(14.4)$ & $113(85.6)$ & \\
\hline \multicolumn{4}{|l|}{ VDRL of mother } \\
\hline Negative & $4(22.2)$ & $14(77.8)$ & \multirow{2}{*}{0.333} \\
\hline Not tested & $16(12.2)$ & $115(87.8)$ & \\
\hline \multicolumn{4}{|l|}{ Mode of delivery } \\
\hline SVD & $9(11.4)$ & $70(88.6)$ & \multirow{3}{*}{0.961} \\
\hline CS & $9(16.7)$ & $45(83.3)$ & \\
\hline Other (eg., breech, vacuum) & $2(12.5)$ & $14(87.5)$ & \\
\hline
\end{tabular}

VDRL $=$ Venereal Disease Research Laboratory (test) $S V D=$ Spontaneous vertex delivery $\mathrm{CS}=$ Caesarean section

Figure 4: Daily bilirubin levels in neonates with severe and clinical neonatal jaundice

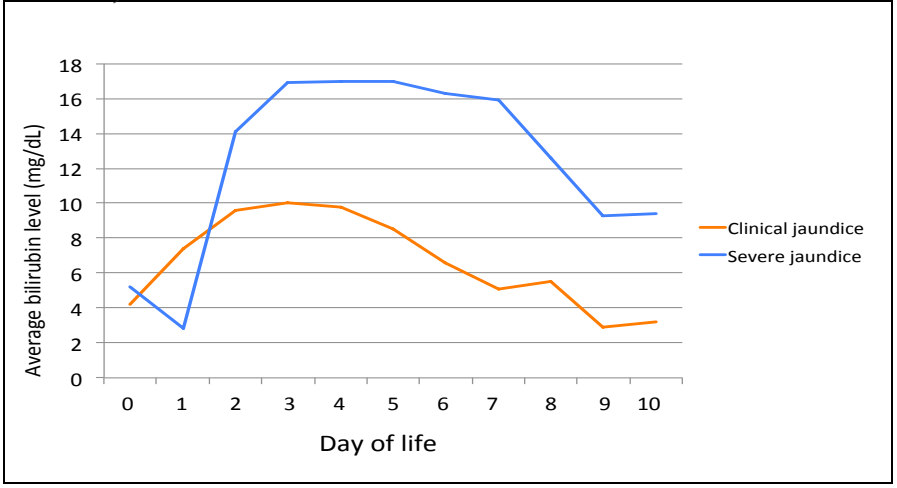

Table 5: Estimated odds ratios from the logistic regression model for clinical jaundice and several predictor variables

\begin{tabular}{lccc}
\hline Variable & Odds ratio & $\begin{array}{c}95 \% \mathrm{Cl} \text { for odds } \\
\text { ratio }\end{array}$ & p-value \\
\hline Birth weight & 0.32 & $0.07-1.46$ & 0.140 \\
Caesarean section & 0.34 & $0.07-1.63$ & 0.175 \\
Gestational age & 1.27 & $0.29-5.47$ & 0.753 \\
Gender & 2.62 & $1.08-6.37$ & 0.033 \\
HIV status & 0.10 & $0.03-0.35$ & $\mathrm{p}<0.001$ \\
SVD & 1.19 & $0.24-5.83$ & 0.834 \\
VDRL status & 0.97 & $0.26-3.68$ & 0.969 \\
\hline
\end{tabular}

SVD $=$ Spontaneous vertex delivery

VDRL $=$ Venereal Disease Research Laboratory (test)

Table 6: Estimated odds ratios from the logistic regression model for severe jaundice and several predictor variables

\begin{tabular}{lccc}
\hline Variable & Odds ratio & $\begin{array}{c}95 \% \mathrm{Cl} \text { for odds } \\
\text { ratio }\end{array}$ & p-value \\
\hline Birth weight & 1.72 & $0.32-9.11$ & 0.525 \\
Caesarean section & 1.37 & $0.25-7.48$ & 0.714 \\
Gestational age & 0.52 & $0.10-2.67$ & 0.432 \\
Gender & 2.59 & $0.80-8.42$ & 0.114 \\
HIV status & 0.38 & $0.05-3.22$ & 0.378 \\
SVD & 1.14 & $0.20-6.41$ & 0.880 \\
VDRL status & 1.90 & $0.52-7.01$ & 0.333 \\
\hline
\end{tabular}

SVD = Spontaneous vertex delivery

$\mathrm{VDRL}=$ Venereal Disease Research Laboratory (test)

\section{Discussion}

Our study showed a strong negative association between the incidence of clinical jaundice and HIV exposure. Contrary to our initial hypothesis, the incidence of neonatal jaundice at $\mathrm{CN}$ was less in the HIV-exposed than in the HIV-nonexposed group. There was no association between HIV exposure and severe jaundice.

Studies have found high rates of prematurity, low birth weight, and very low birth weight in the infants of HIVpositive mothers. ${ }^{8,9}$ Notably, prematurity and low birth weight are risk factors that predispose HIV-exposed neonates to develop jaundice. The incidence of neonatal jaundice at $\mathrm{CN}$ was, however, less in the HIV-exposed than in the HIV nonexposed babies, and several explanations could be suggested for the results obtained.

First, it could be because of the relatively small sample size of the study. There were fewer HIV-exposed neonates admitted to $\mathrm{CN}$ than we initially expected. This could be because most HIV-exposed neonates who were born at QECH did not meet the criteria to be admitted to $\mathrm{CN}$. Consequently, the number of HIV-exposed neonates was small and this affected our statistical analysis. The results cannot be generalised to the population.

Additionally, many of the HIV-exposed neonates who were admitted to $\mathrm{CN}$ were of low birth weight and premature, did not have other co-morbidities and were discharged within two days after admission. Bilirubin levels generally peaked on days 3 and 4 (Figure 2), but since most HIV-exposed neonates stayed at $\mathrm{CN}$ for less than 2 days, the results show 
that they did not develop jaundice. However, these HIVexposed neonates might have developed jaundice after discharge and presented to health centres elsewhere.

Furthermore, controlling for confounders using logistic regression, gender and maternal HIV status were negatively and significantly associated with clinical jaundice. This agrees with previous research done elsewhere, which showed that male gender is a known risk factor for pathological jaundice. ${ }^{13}$ Birth weight, gestational age, and mode of delivery were not significantly associated with jaundice.

A possible hypothesis as to why HIV-exposed neonates were less likely to develop jaundice, at least in the initial days of life, might be related to the Malawi's current PMTCT protocol. The national PMTCT guidelines stipulate that all HIV-seroreactive pregnant and breastfeeding women should be started on an antiretroviral therapy regimen that contains efavirenz (along with tenofovir and lamivudine). The efavirenz may act as a foetal liver enzyme inducer, and this would help in the conjugation of bilirubin, resulting in HIV-exposed neonates having less serum bilirubin levels than their HIV-non-exposed counterparts.

\section{Limitations}

The study used a non-invasive technique (transcutaneous bilirubinometer) for measuring the bilirubin levels. These devices are not always accurate, especially if the neonate has been placed under phototherapy. Using the gold standardserum birubin level measurement-would have been more ideal.

The number of neonates who developed jaundice could be higher than that found in this study because most neonates developed jaundice after being discharged from the ward, and this depended on the mothers noticing that their child was looking yellowish.

Lastly, The sample size for HIV-exposed infants was not reached, as we recruited only 17 of the calculated 69 neonates. The study period could have been increased, or the study field could have been extended to other health facilities in order to have had a larger sample size. With a larger sample size, our results could have, at least, approached generalisability on a national level.

\section{Conclusions}

This short cohort study showed that, in young neonates, jaundice was less common in HIV-exposed babies despite the use of nevirapine. Although this is reassuring, a larger study with longer follow-up is needed.

\section{Acknowledgements}

We would like thank Dr. Sarah White, Dr. John Phuka, Mr. Andrew Kumitawa, and Mr. H.E. Misiri for their help with the statistics. Special thanks, also, to University of Malawi College of Medicine and National Commission for Science and Technology (NCST) for providing the funds needed for the study. Most of all, we would like to thank the doctors and nurses at Chatinkha Nursery, Queen Elizabeth Central Hospital for assistance throughout the data collection period.

\section{References}

1. Israel-Aina YT, Omoigberale AI. Risk factors for neonatal jaundice in babies presenting at the University of Benin Teaching Hospital, Benin City. Niger J Paed. 2012 Mar;39:159-63.

2. Kliegman RM, Behrman RE, Jenson HB, Stanton BF, editors. Nelson textbook of pediatrics. 18th ed. Philadelphia: Saunders Elsevier; 2007.

3. Lissauer T, Clayden G, editors. Illustrated textbook of paediatrics. 2nd ed. London: Mosby; 2001.

4. South Central Network Quality Care Group. Guideline framework for treating neonatal jaundice with phototherapy [Internet]. National Health Service South Central; 2012 [cited 2014 Mar 24]. Available from: https://www.networks.nhs.uk/nhs-networks/thames-valleywessex-neonatal-network/documents/guidelines

5. World Health Organisation. Pocket book of hospital care for children: guidelines for the management of common illnesses with limited resources. Geneva: World Heath Organization; 2005.

6. Tikmani SS, Warraich HJ, Abbasi F, Rizvi A, Darmstadt GL, Zaidi AK. Incidence of neonatal hyperbilirubinemia: a populationbased prospective study in Pakistan. Trop Med Int Health. 2010 May;15(5):502-7. doi: 10.1111/j.1365-3156.2010.02496.x.

7. World Health Organization, United Nations Children's Funds, Joint United Nations Programme on HIV AIDS. Global HIV/AIDS response: epidemic update and health sector progress towards universal access: progress report. Geneva: World Health Organization; 2011.

8. Martin R, Boyer P, Hammil H, Peavy H, Platzker A, Settlage R, et. al. Incidence of premature birth and neonatal respiratory disease in infants of HIV-positive mothers. J Pediatr. 1997 Dec;131(6):851-6.

9. Lepage P, Dabis F, Hitimana DG, Msellati P, Van Goethem C, Stevens AM et al. Perinatal transmission of HIV-1: lack of impact of maternal HIV infection on characteristics of livebirths and on neonatal mortality in Kigali, Rwanda. AIDS. 1991 Mar;5(3):295-300.

10. Ministry of Health, Malawi. Clinical management of HIV in children and adults: Malawi integrated guidelines for providing HIV services in antenatal care, maternity care, under 5 clinics, family planning clinics, exposed infant/pre-ART clinics, ART clinics [Internet]. Lilongwe: Ministry of Health, Malawi; 2011 [cited 2014 Mar 24]. 79 p. Available from: http://www.who.int/hiv/pub/guidelines/malawi_art.pdf.

11. Ocama P, Katwerere M, Piloya T, Feld J, Opio KC, Kambuju A, et al. The spectrum of liver diseases in HIV infected individuals at an HIV treatment clinic in Kampala, Uganda. Afr Health Sci. 2008 Mar;8(1):812

12. Tanja L, Routh J, Kennedy N, Jefferis O, Stott P, editors. Protocols for the management of common childhood illnesses in Malawi. Blantyre: Department of Paediatrics \& Child Health, Queen Elizabeth Central Hospital; 2011.

13. Maisels MJ, Clifford K, Antle CE, Leib GR. Jaundice in the healthy newborn infant: a new approach to an old problem. Pediatrics. 1988 Apr;81(4):505-11. 